Intensivmed 2010 • 47:224-224

DOI 10.1007/s00390-009-0154-9

Online publiziert: 30 . April 2010

(c) Springer-Verlag 2010

\author{
H. Messmann \\ III. Medizinische Klinik, Klinikum Augsburg
}

\title{
Intensiv- und Notfallmedizin in der Gastroenterologie
}

entweder aufgrund ihrer schweren Grunderkrankung, wie z. B. beim Olgivie-Syndrom, oder aber weil lebenswichtige Medikamente zu bedrohlichen Nebenwirkungen seitens des Gastrointestinaltraktes führen. Ein weiteres Problem stellen nosokomiale gastrointestinale Blutungen dar und hier insbesondere gastrointestinale Blutungen beim Intensivpatienten (F. Klebl). Welche Stressulkusprophylaxe soll es sein? Wer braucht sie und wer nicht? Hier warten wir seit Jahren vergebens auf die Empfehlungen der entsprechenden Fachgesellschaften. wenn wir uns zunehmend spezialisieren, so muss es unser Ziel als Internisten sein, als sog. „Allrounder“ oder „Generalisten“ der Inneren Medizin die akut- und notfallmedizinischen Aspekte in unserem Fachgebiet kompetent zu vertreten.

Mit diesem Heft möchte ich dazu beitragen, dass all diejenigen, die gerade nicht schwerpunktmäßig gastroenterologisch tätig sind, bei ihrer täglichen Arbeit auf der Intensivstation oder in der Notaufnahme vom Inhalt dieser Beiträge profitieren.

Das „akute Abdomen“ in der Notaufnahme (M. Reng) ist ein Paradebeispiel dafür, wie man interdisziplinär zusammenarbeiten muss und was unter heutigem Kostendruck eine rationelle Diagnostik bedeutet.

Sowohl beim akuten Leberversagen (C. Trautwein) als auch bei der akuten Pankreatitis (W. Huber) handelt es sich um schwere, oftmals lebensbedrohliche Erkrankungen, bei der der Intensivmediziner als Gastroenterologe oder der Gastroenterologe als Intensivmediziner gefordert ist.

Eine Reihe von Krankheitsbildern tritt aber oftmals begleitend bei Intensivpatienten auf. Eine Vielzahl von Patienten leidet an Motilitätsstörungen (H. Allescher)

\section{( Intensivmedizin ist und bleibt eine große Herausforderung in unserem Gesundheitssystem}

Die akute mesenteriale Ischämie des Gastrointestinaltraktes (G. Lock) stellt mit zunehmendem Alter der Patienten ein immer häufigeres Krankheitsbild dar und ist mit einer hohen Mortalität assoziiert. Hinzu kommt, dass viele Intensivpatienten zusätzlich zu ihren schweren Grunderkrankungen ein hohes Risiko haben, aus vielerlei Gründen an einer akuten Ischämie zu erkranken.

Ein weiteres, immer größeres Problem stellt der dramatische Anstieg an Infektionen mit Clostridium perfringens dar. Das Krankheitsbild der pseudomembranösen Kolitis (B. Salzberger) hat längst Einzug auf unseren Intensivstationen gefunden und fordert von uns Medizinern einmal mehr, dass wir durch rationellen und konsequenten Einsatz von Antibiotika auch präventiv handeln müssen.

Der Stellenwert der Ernährung von Intensivpatienten (W. Druml) hat sich in den letzten Jahren grundlegend geändert. Ihr kommt zunehmend auch ein präven- tiver und therapeutischer Aspekt zu, und das nicht nur bei der akuten Pankreatitis.

Intensivmedizin ist und bleibt eine große Herausforderung in unserem $\mathrm{Ge}$ sundheitssystem. Nicht alles Machbare ist sinnvoll, und nicht alles Sinnvolle ist machbar. Ethische Fragen auf der einen Seite und Kostendruck auf der anderen Seite bestimmen längst unser tägliches Handeln auf den Intensivstationen. Die Basis für eine patientengerechte intensivund notfallmedizinische Versorgung sind eine optimale Ausbildung unseres medizinischen Nachwuchses sowie die fach- und berufsgruppenübergreifende Diskussion.

Ich wünsche Ihnen viel Spaß beim Lesen und würde mich sehr freuen, wenn ich Sie alle zur 42. Gemeinsamen Jahrestagung der Deutschen Gesellschaft für Internistische Intensivmedizin und Notfallmedizin und der Österreichischen Gesellschaft für Internistische und Allgemeine Intensivmedizin in Berlin begrüßen dürfte.

Ich freue mich jetzt schon auf interessante und spannende Tage mit Ihnen zusammen in Berlin.

Herzliche Grüße

Ihr
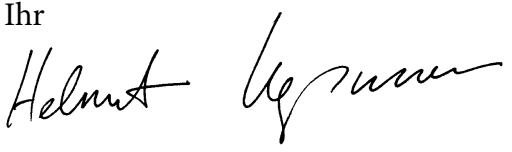

Helmut Messmann

\section{Korrespondenzadresse \\ Prof. Dr. H. Messmann}

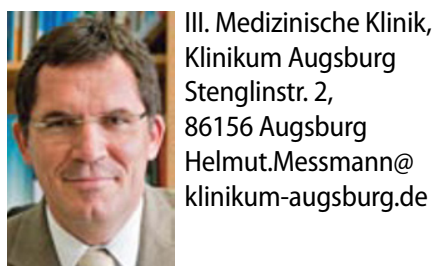

